\title{
SCIENTIFIC REPORTS

\section{Factors associated with platelet reactivity during dual antiplatelet therapy in patients with diabetes after acute coronary syndrome}

\author{
Vacis Tatarunas ${ }^{1 *}$, Nora Kupstyte-Kristapone ${ }^{1,2,3}$, Vaidotas Zvikas ${ }^{4}$, Valdas Jakstas ${ }^{4}$, \\ Remigijus Zaliunas $^{2}$ \& Vaiva Lesauskaite ${ }^{1}$
}

Antiplatelet drugs are prescribed without considering the diabetic status of the patient. The objective of the current investigation was to determine the impact of clinical factors, CYP4F2 enzyme and 20-hydroxyeicosatetraenoic acid (20-HETE) concentrations on high on-treatment platelet reactivity in patients with diabetes treated with antiplatelet drugs following acute coronary syndromes. A total of 667 patients were included in the study. Dual antiplatelet drug loading dosages with aspirin $(300 \mathrm{mg})$ and ticagrelor $(180 \mathrm{mg})$ or clopidogrel $(600 \mathrm{mg})$ were prescribed to all the studied patients. Testing of platelet aggregation was performed the day after loading antiplatelet drug dosages. Platelet aggregation test was done according to the classical Born method. Multivariate binary regression analysis demonstrated that insulin use and higher 20-HETE concentration increased the odds of high on-treatment platelet reactivity during the initiation of antiplatelet drug therapy (OR: $3.968,95 \% \mathrm{Cl}$ : 1.478-10.656, $p=0.006$ and OR: $1.139,95 \% \mathrm{Cl}: 1.073-1.210$, respectively, $p<0.001$ ). Ticagrelor use decreased the odds of developing high on-treatment platelet reactivity (OR: $0.238,95 \% \mathrm{Cl}: 0.097-$ $0.585, p=0.002$ ). Data from this study revealed that high on-treatment platelet reactivity during dual antiplatelet therapy in patients with diabetes may depend on such factors as insulin prescription and 20-HETE concentration.

Diabetes may enhance the effect of risk factors involved in cardiovascular disease ${ }^{1}$. However, the treatment guidelines for acute coronary syndrome (ACS) recommend the same treatment strategy for both diabetic and non-diabetic patients ${ }^{1,2,3}$. Antiplatelet drugs are prescribed without considering the diabetic status of the patient ${ }^{2}$.

In the PLATelet inhibition and patient Outcomes (PLATO) study, the newer generation drug ticagrelor was demonstrated to be superior to clopidogrel in reducing ischaemic events in ACS patients, irrespective of whether they had diabetes ${ }^{4}$. More recent studies have reported that patients treated with clopidogrel had higher in-hospital mortality compared to those treated with prasugrel or ticagrelor ${ }^{5}$. Platelet aggregation studies also showed that patients with diabetes who use clopidogrel have higher platelet reactivity than patients without diabetes ${ }^{6}$. A critical meta-analysis done with 26 studies, which included 28.178 patients, recommended platelet reactivity testing in high risk patients (patients with diabetes, patients with multiple cardiovascular risk factors, and in patients with comorbidities $)^{7}$.

Higher platelet reactivity in diabetic patients is due to increased platelet functions, including an increased response to stimulation by platelet aggregation agonists, adhesion to thrombogenic surfaces and platelet aggregation $^{8}$. In general, oxidative stress and reduced antioxidant activity induced by hyperglycaemia significantly augment in diabetic patients, subsequently leading to platelet activation and hyperreactivity ${ }^{9}$. A recent study by Tunaru et al. showed that diabetes is also a disease of fatty acid metabolism ${ }^{10}$. Tunaru et al. showed that glucose induces the synthesis of 20-hydroxyeicosatetraenoic acid $(20 \text {-HETE })^{10} .20$-HETE is a CYP-derived eicosanoid, which is produced by CYP4A11 and CYP4F2 enzymes, usually found in the kidneys and the liver ${ }^{11,12}$. According

\footnotetext{
${ }^{1}$ Institute of Cardiology of Lithuanian University of Health Sciences, Sukileliu 15, Kaunas, LT, 50009, Lithuania. ${ }^{2}$ Department of Cardiology of Lithuanian University of Health Sciences, Eiveniu 2, LT, 50009, Kaunas, Lithuania. ${ }^{3}$ Cardiovascular Center of Republican hospital of Siauliai, V. Kudirkos g. 99, 76231, Siauliai, LT, Lithuania. ${ }^{4}$ Institute of Pharmaceutical Technologies of Lithuanian University of Health Sciences, Sukileliu 13, Kaunas, LT, 50009, Lithuania. *email: vacis.tatarunas@lsmuni.lt
} 


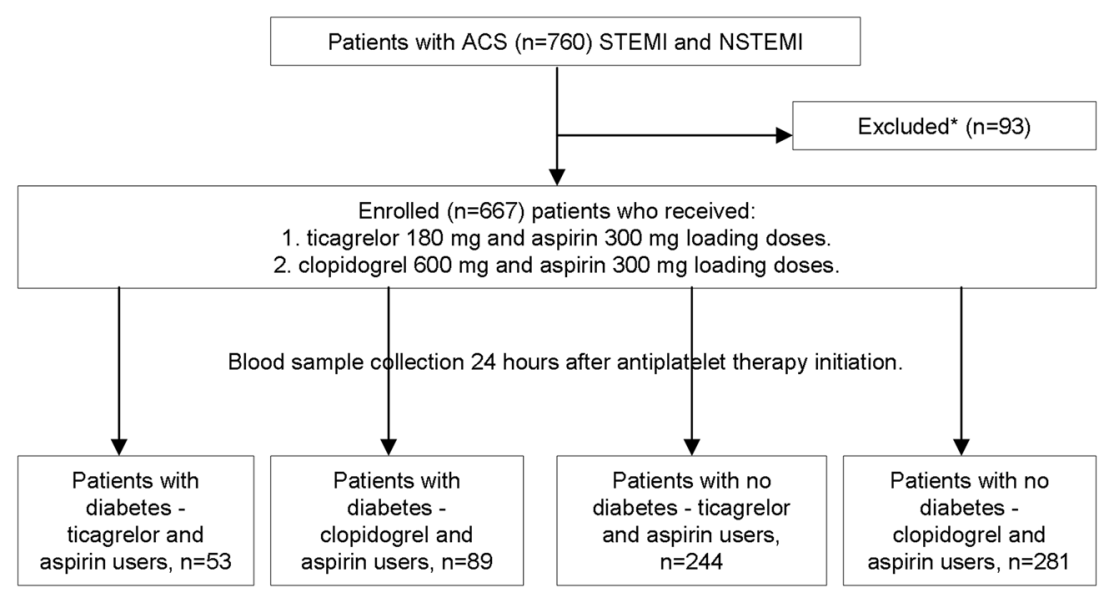

Figure 1. Study design. *Inclusion and exclusion criteria described in materials and methods section.

to Tunaru et al., reduced 20-HETE formation leads to reduction of glucose-stimulated insulin secretion in pancreatic islets ${ }^{10}$.

Previous studies by our group showed that CYP4F2 variants may significantly affect antiplatelet therapy ${ }^{13-15}$. However, we were unable to find a relationship of plasma CYP4F2 enzyme activity and 20-HETE concentrations on antiplatelet activity of drugs (in a relatively small sample of the patients, $n=146$ ) during dual antiplatelet therapy (DAPT) ${ }^{15}$.

\section{Methods}

The aim of the current investigation was to analyse the impact of clinical factors, CYP4F2 and 20-HETE concentrations on high on-treatment platelet reactivity in a sample of the patients representing a significant group of patients with diabetes treated with antiplatelet drugs ticagrelor or clopidogrel following acute coronary syndromes.

Study population and inclusion criteria. Clinical data and DNA samples of the patient population were collected during the SEN-09/2015 study ${ }^{14}$. All the patients were hospitalised for percutaneous coronary interventions (PCI) and stent implantation due to acute coronary syndromes (myocardial infarction or unstable angina) at the Department of Cardiology at the Lithuanian University of Health Sciences (LUHS) in Kaunas, Lithuania, from 2013 to 2017. Patient inclusion and exclusion criteria were described earlier ${ }^{14}$. Patients were divided into four groups according to their diabetic status (diabetic or non-diabetic) and the prescribed antiplatelet drug (Fig. 1): diabetic patients who were prescribed ticagrelor and aspirin, diabetic patients who were prescribed clopidogrel and aspirin, non-diabetic patients who were prescribed ticagrelor and aspirin, and non-diabetic patients who were prescribed clopidogrel and aspirin.

Patient clinical data. A total of 667 patients were included in the study (Fig. 1). Dual antiplatelet drug loading dosages with aspirin $(300 \mathrm{mg})$ and ticagrelor $(180 \mathrm{mg})$ or clopidogrel $(600 \mathrm{mg})$ were prescribed to patients before PCI, according to the European Society of Cardiology (ESC) guidelines ${ }^{16}$. Patients were prescribed either clopidogrel or ticagrelor irrespective of diabetes. Blood samples for the analysis were taken the next day, approximately 24 hours after loading of antiplatelet dosages.

All patients received standard treatment with statins, angiotensin-converting enzyme inhibitors (or angiotensin receptor I blockers) and $\beta$-adreno-blockers. Clinical characteristics of the patients were collected from the case histories.

Platelet aggregation. Testing of platelet aggregation was performed as described above ${ }^{15}$, the next day after initiation of antiplatelet therapy. Classical Born method was used to perform platelet aggregation test. The intensity of light transmission in platelet rich and in platelet poor plasma was compared by using Chrono-Log 4902D platelet aggregometer (Chrono-Log, Havertown, Pennsylvania, USA). Platelet aggregation was determined following induction with adenosine diphosphate (ADP; final concentration $5 \mu \mathrm{M}$; Chrono-Log, Havertown, Pennsylvania, USA), epinephrine (final concentration $10 \mu \mathrm{M}$; Chrono-Log, Havertown, Pennsylvania, USA) and arachidonic acid (ARA) (final concentration $0.5 \mathrm{mM}$; Chrono-Log, Havertown, Pennsylvania, USA).

Detection of CYP4F2 and 20-HETE in blood plasma. Concentrations of the CYP4F2 enzyme and 20-HETE in blood plasma were measured as described above ${ }^{15}$. All plasma samples were tested in duplicate. The analysis of 20-HETE in blood plasma was performed at the Institute of Pharmaceutical technologies of the Lithuanian University of Health Sciences. Measurements of CYP4F2 enzyme were performed at the certified Laboratory of Molecular Cardiology. The microplate reader Stat-fax 4200 (Awareness Technology, Palm City, Florida, USA) with a primary wave-length of $450 \mathrm{~nm}$ was used. The intraassay precision of the CYP4F2 SEL399Hu kit is less than $10 \%$, and the interassay precision is less than $12 \%$ as declared by the manufacturer of this kit. No significant cross-reactivity or interference between CYP4F2 and analogues was declared by the manufacturer. A group of healthy $(n=26)$ subjects (age range 29-90, median 54 years), consisting of 13 males and 
13 females who did not have complains about their personal health, and were not taking antibiotics, antiplatelets and anticoagulants within the past 3 months, was also gathered in order to determine blood plasma CYP4F2 and 20-HETE concentration in healthy subjects.

Statistical analysis. Platelet aggregation and concentrations of CYP4F2 and 20-HETE in blood plasma were evaluated using a nonparametric Kruskal-Wallis test. Fisher's exact test and Pearson $\chi 2$ analysis were used for categorical variables. The relationship between the high on-treatment platelet reactivity after induction with ADP and patient clinical data was established using multivariate linear regression analysis. High on-treatment platelet reactivity was defined as platelet aggregation levels higher than $46 \%$ after induction with $\mathrm{ADP}^{17}$. All variables were chosen for the multivariable model by backward selection, with the final model containing only those with $\mathrm{p}<0.05$.

Ethics approval and consent to participate. All the procedures used have been reviewed in compliance with the ethical standards of the Regional Bioethics Committee of Kaunas, Lithuania (the permission number is BE-2-42) and the World Medical Association Declaration of Helsinki on Ethical Principles for Medical Research Involving Human Subjects. Written informed consent was obtained from all the participants prior to inclusion in the study.

\section{Results}

Among the patients, clopidogrel users were older as compared to ticagrelor users (range 39-89, median 68 years vs. range 29-90, median 64 years, $p=0.001$ ). Patients with diabetes tended to receive clopidogrel more often than ticagrelor $(24.1 \%$ vs $17.8 \%, p=0.08)$. Also, patients with diabetes tended to be older than non-diabetic patients (range 37-86, median 69 years vs. range 29-90, median 66 years, $p=0.053$ ). Female patients with diabetes received clopidogrel more often than non-diabetic female patients (Table 1). Female patients with diabetes that used clopidogrel were older than ticagrelor users $(\mathrm{p}=0.043)$. Patients with diabetes had bigger body weight, body weight index and body waist circumference (Table 1). More non-diabetic patients with ST segment elevation myocardial infarction (STEMI) were prescribed ticagrelor. More patients with diabetes and recurrent myocardial infarction (reMI) were prescribed clopidogrel. Patients with diabetes were less frequently smokers (Table 1).

Calcium channel blockers were less frequently prescribed to ticagrelor users and non-diabetic patients. Patients with diabetes that used clopidogrel had higher creatinine levels and more frequently received diuretics.

Users of ticagrelor had higher platelet (range 103-1000, median $227 \times 10^{9} / 1$ vs. range $94-796$, median $211 \times 10^{9} / 1, p=0.001$ ) and white blood cell (range $3-43$, median $9 \times 10^{9} / 1$ vs. range $4-28$, median $8 \times 10^{9} / 1$, $p=0.012$ ) counts when compared to clopidogrel users.

Effect of antiplatelet activity in diabetic and non-diabetic patients. Platelet aggregation values with ADP were higher in clopidogrel users than in ticagrelor users (range 5-70, median 33\% Agr vs. range 5-77, median $\left.20 \%{ }^{\mathrm{Agr}}, p<0.001\right)$. Higher platelet aggregation values were detected in diabetic patients who were prescribed clopidogrel compared to non-diabetic patients $(p<0.001)$, (Table 2$)$. No such effect was found in ticagrelor users.

The impact of CYP4F2 and 20-HETE concentration in blood plasma on antiplatelet activity during initiation of dual antiplatelet therapy. Control sample with healthy subjects showed higher CYP4F2 concentrations in healthy subjects than in patients: range $6-81$, median $15 \mathrm{ng} / \mathrm{ml}$ vs. range $0-132$, median $9 \mathrm{ng} /$ $\mathrm{ml}, p<0.001$, respectively. Levels of 20-HETE did not significantly differ in patients and in healthy subjects: range $1-30$, median $6 \mathrm{ng} / \mathrm{ml}$ vs range $2-11$, median $5 \mathrm{ng} / \mathrm{ml}, \mathrm{p}=0.133$.

The CYP4F2 concentration was lower in clopidogrel users compared to ticagrelor users (range 0-132, median $5 \mathrm{ng} / \mathrm{ml}$ vs. range $0-101$, median $12 \mathrm{ng} / \mathrm{ml}, p<0.001$ ). The CYP4F2 concentration (Table 2) was lower in non-diabetic patients that used clopidogrel compared to patients with diabetes $(p=0.037)$. Patients with diabetes, ticagrelor users tended to have higher CYP4F2 concentration than non-diabetic patients (Table 2).

Levels of 20-HETE were higher in patients with diabetes who were prescribed ticagrelor compared to non-diabetic patients (Table 2).

Patients with high on-treatment platelet reactivity had a lower CYP4F2 (range 0-122, median $6 \mathrm{ng} / \mathrm{ml}$ vs. range $0-132$, median $9 \mathrm{ng} / \mathrm{ml}, p=0.019$ ), but a higher 20 -HETE (range $1-28$, median $10 \mathrm{ng} / \mathrm{ml}$ vs. range $1-30$, median $6 \mathrm{ng} / \mathrm{ml}, p<0.001)$ concentration than patients with a good response to antiplatelet therapy.

Binary logistic regression model. Multivariate binary regression analysis (Table 3) demonstrated that insulin use and a higher 20-HETE concentration all increased the odds of high on-treatment platelet reactivity during the initiation of antiplatelet therapy. Ticagrelor use decreased the risk of developing high on-treatment platelet reactivity (Table 3 ).

\section{Discussion}

The results of this study demonstrated that CYP4F2 and 20-HETE may have a significant role in patients with diabetes and in users of dual antiplatelet therapy. For the first time, the results revealed that patients with high on-treatment platelet reactivity had a higher 20-HETE but a lower CYP4F2 concentration in blood plasma. Patients users of clopidogrel had a lower CYP4F2 concentration than ticagrelor users. Healthy subjects had a higher CYP4F2 concentration than our studied patients. Also, patients with diabetes, users of ticagrelor, had a higher 20-HETE concentration than non-diabetic patients.

Despite research data showing decreased activity of clopidogrel in diabetics, there are currently no guidelines which recommend different strategies for patients with diabetes ${ }^{2,3}$. Patients in the present study who were prescribed ticagrelor were younger and more frequently had STEMI. Patients with recurrent MI (in anamnesis) and 


\begin{tabular}{|c|c|c|c|c|c|c|}
\hline & \multicolumn{2}{|c|}{ Ticagrelor users } & \multirow[b]{2}{*}{$p$} & \multicolumn{2}{|c|}{ Clopidogrel users } & \multirow[b]{2}{*}{$p$} \\
\hline & Diabetes & No diabetes & & Diabetes & No diabetes & \\
\hline & $53(100)$ & $244(100)$ & & $89(100)$ & $281(100)$ & \\
\hline \multicolumn{7}{|l|}{ Gender n (\%) } \\
\hline Males & $42(79.2)$ & $179(73.4)$ & 0.487 & $54(60.7)$ & $202(71.9)$ & 0.049 \\
\hline Females & $11(20.8)$ & $65(26.6)$ & & $35(39.3)$ & $79(28.1)$ & \\
\hline $\begin{array}{l}\text { Age in years median (min- } \\
\text { max) }\end{array}$ & $64(38-82)$ & $64(29-90)$ & 0.744 & $69(40-86)$ & $68(39-89)$ & 0.059 \\
\hline Males & $63(38-81)$ & $62(29-90)$ & 0.167 & $66(40-84)$ & $63(39-89)$ & 0.26 \\
\hline Females & $69(43-82)$ & $71(43-88)$ & 0.218 & $74(60-86)$ & $72(56-88)$ & 0.513 \\
\hline $\begin{array}{l}\text { Patient weight in } \mathrm{kg} \text { median } \\
\text { (min-max) }\end{array}$ & $95(60-121)$ & $84(48-164)$ & $<0.001$ & $92(47-158)$ & $82(45-132)$ & $<0.001$ \\
\hline $\begin{array}{l}\begin{array}{l}\text { Body weight index median } \\
\text { (min-max) }\end{array} \\
\end{array}$ & $31(23-43)$ & $28(17-44)$ & $<0.001$ & $32(16-50)$ & $28(17-55)$ & $<0.001$ \\
\hline $\begin{array}{l}\text { Waist circumference median } \\
(\min -\max )\end{array}$ & $99(81-130)$ & $94(63-122)$ & 0.001 & $104(68-137)$ & $93(69-128)$ & $<0.001$ \\
\hline \multicolumn{7}{|l|}{ ST-elevation MI n (\%) } \\
\hline STEMI & $16(30.2)$ & $119(48.8)$ & 0.015 & $28(31.5)$ & $88(31.3)$ & 1 \\
\hline NSTEMI & $37(69.8)$ & $125(51.2)$ & & $61(68.5)$ & $193(68.7)$ & \\
\hline \multicolumn{7}{|l|}{ MI in anamnesis $\mathrm{n}(\%)$} \\
\hline First & $33(62.3)$ & $168(68.9)$ & 0.418 & $56(62.9)$ & $212(75.4)$ & 0.029 \\
\hline Recurrent & $20(37.7)$ & $76(31.1)$ & & $33(37.1)$ & $69(24.6)$ & \\
\hline \multicolumn{7}{|l|}{ Smoking n (\%) } \\
\hline Smokers & $9(17)$ & $75(30.1)$ & 0.045 & $17(19.1)$ & $87(31)$ & 0.031 \\
\hline Non-smokers & $44(83)$ & $169(69.9)$ & & $72(80.9)$ & $194(69)$ & \\
\hline \multicolumn{7}{|l|}{ Concomitant drug users } \\
\hline Diaprel n (\%) & $8(15.1)$ & - & - & $22(24.7)$ & - & - \\
\hline Glimepiride n (\%) & $3(5.7)$ & - & - & $2(2.2)$ & - & - \\
\hline Insulin n (\%) & $19(35.8)$ & - & - & $34(38.2)$ & - & - \\
\hline Metformin n (\%) & $33(62.3)$ & - & - & $60(67.4)$ & - & - \\
\hline ACE inhibitors n (\%) & $47(88.7)$ & $232(95.1)$ & 0.105 & $79(88.8)$ & $250(88.9)$ & 1 \\
\hline \multicolumn{7}{|l|}{ Angiotensin II } \\
\hline receptor antagonists n (\%) & $5(9.4)$ & $11(4.5)$ & 0.175 & $12(13.5)$ & $28(10)$ & 0.335 \\
\hline \multicolumn{7}{|l|}{ Beta } \\
\hline adrenoblockers n (\%) & $50(94.3)$ & $229(93.9)$ & 1 & $86(96.6)$ & $262(93.2)$ & 0.309 \\
\hline Statins n (\%) & $53(100)$ & $242(99.2)$ & 1 & $84(94.4)$ & $274(97.5)$ & 0.17 \\
\hline Spironolactone n (\%) & $12(22.6)$ & $60(24.6)$ & 0.86 & $23(25.8)$ & $62(22.1)$ & 0.472 \\
\hline Amiodarone n (\%) & $3(5.7)$ & $5(2)$ & 0.155 & $1(1.1)$ & $6(2.1)$ & 1 \\
\hline Ivabradin n (\%) & $7(13.2)$ & $24(9.8)$ & 0.46 & $20(22.5)$ & $42(14.9)$ & 0.105 \\
\hline \multicolumn{7}{|l|}{ Calcium Channel } \\
\hline Blockers n (\%) & $8(15.1)$ & $11(4.5)$ & 0.009 & $11(12.4)$ & $21(7.5)$ & 0.192 \\
\hline Diuretics n (\%) & $20(37.7)$ & $50(20.5)$ & 0.012 & $41(46.1)$ & $71(25.3)$ & $<0.0001$ \\
\hline Ranitidine n (\%) & $35(66)$ & $185(75.8)$ & 0.166 & $59(66.3)$ & $197(70.1)$ & 0.52 \\
\hline Proton pump inhibitors n (\%) & $7(13.2)$ & $18(7.4)$ & 0.175 & $2(2.2)$ & $5(1.8)$ & 0.675 \\
\hline \multicolumn{7}{|l|}{ Blood test parameters } \\
\hline Platelet count $\left(\times 10^{9} / \mathrm{l}\right)$ & $210(109-491)$ & $228(103-1000)$ & 0.09 & $213(94-422)$ & $211(101-796)$ & 0.692 \\
\hline WBC $\left(\times 10^{9} / 1\right)$ & $9(4-43)$ & $9(3-22)$ & 0.788 & $9(4-21)$ & $8(4-28)$ & 0.206 \\
\hline C-reactive protein $(\mathrm{mg} / \mathrm{l})$ & $9(1-276)$ & $4(1-379)$ & 0.115 & $5(1-222)$ & $4(1-255)$ & 0.064 \\
\hline Creatinine $(\mu \mathrm{mol} / \mathrm{l})$ & $94(53-262)$ & $89(53-303)$ & 0.154 & $92(47-478)$ & $87(48-220)$ & 0.016 \\
\hline Hemoglobin $(\mathrm{g} / \mathrm{l})$ & \begin{tabular}{|l|l}
$132(98-176)$ \\
\end{tabular} & $136(89-172)$ & 0.437 & $137(81-163)$ & \begin{tabular}{|l|}
$139(50-170)$ \\
\end{tabular} & 0.143 \\
\hline
\end{tabular}

Table 1. Baseline patients' sample description. WBC - white blood cells.

diabetes were more frequently prescribed clopidogrel. Patients with diabetes tended to be older than non-diabetic patients. In addition, female patients with diabetes were less frequently prescribed ticagrelor than non-diabetic females. The rate of clopidogrel prescription to diabetic patients observed in our study confirms the observations of other researchers, who emphasise that factors such as diabetes have no decisive significance in clinical practice when choosing a more potent antiplatelet drug than clopidogrel ${ }^{1,18,19}$. Diabetes may, however, aggravate other risk factors involved in cardiovascular disease, especially in women ${ }^{1}$.

It is noteworthy that our presented patients users of ticagrelor had elevated platelet and white blood cells counts. Patients with diabetes users of clopidogrel had higher creatinine level and more frequently received 


\begin{tabular}{|c|c|c|c|c|c|c|}
\hline & \multicolumn{2}{|c|}{ Ticagrelor users } & \multirow[b]{2}{*}{$p$} & \multicolumn{2}{|c|}{ Clopidogrel users } & \multirow[b]{2}{*}{$p$} \\
\hline & $\begin{array}{l}\text { Diabetes } \\
(\mathrm{n}=53)\end{array}$ & $\begin{array}{l}\text { No diabetes } \\
(\mathrm{n}=244)\end{array}$ & & $\begin{array}{l}\text { Diabetes } \\
(n=89)\end{array}$ & $\begin{array}{l}\text { No diabetes } \\
(\mathrm{n}=281)\end{array}$ & \\
\hline $\begin{array}{l}\text { Platelet aggregation } \\
\text { with ADP \% }\end{array}$ & $21(6-68)$ & $20(5-77)$ & 0.186 & $39(9-69)$ & $31(5-70)^{*}$ & $<0.001$ \\
\hline $\begin{array}{l}\text { Platelet aggregation } \\
\text { with epinephrine } \% \text { Agr }\end{array}$ & $29(6-74)$ & $30(4-94)$ & 0.89 & $35(10-92)$ & $34(6-89)$ & 0.62 \\
\hline \multirow{2}{*}{$\begin{array}{l}\text { Platelet aggregation } \\
\text { with ARA \% }{ }^{\text {Agr }}\end{array}$} & $13(6-81)$ & $13(4-77)$ & 0.484 & $14(6-90)$ & $16(4-73)$ & \multirow{2}{*}{0.593} \\
\hline & $(\mathrm{n}=43)$ & $(n=197)$ & & $(\mathrm{n}=83)$ & $(\mathrm{n}=233)$ & \\
\hline \multirow{2}{*}{ CYP4F2 conc. ng/ml } & $14(3-90)$ & $12(0-101)$ & 0.096 & $6(0-88)$ & $4(0-132)$ & \multirow{2}{*}{0.037} \\
\hline & $\mathrm{n}=47$ & $\mathrm{n}=208$ & & $\mathrm{n}=72$ & $\mathrm{n}=226$ & \\
\hline \multirow{2}{*}{ 20-HETE ng/ml } & $8(1-30)$ & $6(1-27)$ & 0.006 & $6(1-24)$ & $6(1-26)$ & \multirow{2}{*}{0.983} \\
\hline & $\mathrm{n}=33$ & $\mathrm{n}=126$ & & $\mathrm{n}=48$ & $\mathrm{n}=118$ & \\
\hline
\end{tabular}

Table 2. Platelet aggregation levels, CYP4F2 and 20-HETE concentrations in diabetic and non-diabetic patients users of ticagrelor and clopidogrel.

\begin{tabular}{|l|l|l|c|}
\hline Variable & $\begin{array}{l}\text { Odds } \\
\text { ratio }\end{array}$ & 95\% CI & \multicolumn{1}{l|}{$\boldsymbol{p}$} \\
\hline Insulin use & 3.968 & $1.478-10.656$ & 0.006 \\
\hline Ticagrelor use & 0.238 & $0.097-0.585$ & 0.002 \\
\hline 20-HETE $(\mathrm{ng} / \mathrm{ml})$ & 1.139 & $1.073-1.210$ & $<0.001$ \\
\hline
\end{tabular}

Table 3. Multivariate binary regression analysis of the association between clinical factors and high ontreatment platelet reactivity.

diuretics. Low lymphocyte count is a poorer prognostic marker during STEMI, as it correlates with inflammatory process during acute myocardial infarction. Low lymphocyte count during first 96 hours of a STEMI might predict recurrent myocardial infarction ${ }^{20}$. Studies also showed that low glomerular filtration rate might be associated with worse outcomes in ACS STEMI and non-ST-segment elevation myocardial infarction (NSTEMI) patients ${ }^{21}$.

The PLATO study, conducted about a decade ago, showed that ticagrelor is more effective for reducing ischaemic events in diabetic patients with ACS when compared to clopidogrel ${ }^{4}$. PLATO study results also showed, that diabetes has no effect on treatment outcomes during ticagrelor therapy ${ }^{4}$. However, Nardin et al., in a small patient sample $(n=224)$, recently showed that diabetic patients who used ticagrelor had a higher rate of platelet reactivity than non-diabetic patients ${ }^{22}$. Our results are in line with those of the PLATO study. Diabetes had no effect on antiplatelet treatment with ticagrelor, but significantly reduced the antiplatelet effect of clopidogrel. Ticagrelor is a newer generation, faster onset antiplatelet drug than clopidogrel. It acts as a reversible non-competitive P2Y12 receptor inhibitor, and binds distinct from the ADP binding site in the P2Y12 receptor. Clopidogrel, on the contrary, binds directly at the binding site of ADP and is its competitive inhibitor. Ticagrelor does not require metabolic activation ${ }^{23,24}$. By contrast, clopidogrel requires metabolic activation, and its therapeutic effect is highly dependent on hepatic cytochrome P450 enzyme activity (mainly CYP2C19). Variants of CYP2C19 have a critical effect during clopidogrel therapy ${ }^{25,26}$. The therapeutic effect of clopidogrel is also influenced by other factors, which do not usually affect treatment with ticagrelor ${ }^{14}$. Ticagrelor has a pleiotropic activity. It inhibits the reuptake of adenosine by red blood cells, thereby elevating blood plasma adenosine concentrations ${ }^{27}$. Elevated adenosine concentrations might reduce platelet aggregation by acting on low-affinity platelet $\mathrm{A}_{2 \mathrm{~A}}$ receptors ${ }^{27}$. In addition, adenosine dilates coronary arterioles in humans ${ }^{28}$.

In our earlier investigations, we found that $C Y P 4 F 2$ gene variants may significantly affect antiplatelet therapy during clopidogrel or ticagrelor treatment after $\mathrm{ACS}^{13,14}$. In the current study, we performed a more profound analysis in a larger sample of patients. Data from the current investigation show higher CYP4F2 concentration in blood plasma of healthy subjects than in patients. Also, similar CYP4F2 concentrations are found in ticagrelor users but are lower in blood plasma of clopidogrel users. Most of cytochromes P450 are suppressed during inflammation by proinflammatory cytokines, eicosanoids and histamine ${ }^{29,30}$. It is noteworthy that CYP4F 2 is usually induced during inflammation ${ }^{31,32}$, as it performs $\omega$-hydrolysis of leukotriene B4 or arachidonic acid ${ }^{33}$, and it plays a critical role in resolution of inflammation ${ }^{31,32}$. Patients with diabetes users of clopidogrel have a higher CYP4F2 concentration than non-diabetic patients. Also, patients with diabetes users of ticagrelor tended to have a higher concentration of CYP4F2 in blood plasma than non-diabetics. Higher concentration of CYP4F2 in patients with diabetes might be associated with higher extent of inflammatory processes as compared to the patients without diabetes. Higher CYP4F2 concentration in plasma of ticagrelor users might also be determined by pleiotropic effects of ticagrelor ${ }^{27}$. Ticagrelor may participate in resolution of inflammation in patients with ACS. However, the first limitation of this study is that we did not evaluate the activity of CYP4F2, as we only detected the concentration of this enzyme. Another limitation is that we did not determine the origin of this enzyme. CYP4F2 is an enzyme usually found in endoplasmic reticulum. However, under stress conditions, it may be secreted with extracellular vesicles into the blood stream ${ }^{34}$.

20-HETE is a metabolite of arachidonic acid, which is formed by hepatic cytochrome P450 w-hydroxylases. The two major enzymes that produce 20-HETE in humans are CYP4F2 and CYP4A1 $1^{35}$. In general, the CYP4F2 
enzyme is usually expressed in the kidney and liver. CYP4A11 is found in the S2 and S3 segments of the proximal renal tubules ${ }^{11,12}$. Our results showed that concentration of 20-HETE was higher in patients with diabetes receiving ticagrelor as compared to non-diabetic patients. The most interesting is that our presented patients with high on-treatment platelet reactivity had elevated 20-HETE, but a lower CYP4F2 concentration. CYP4F2 may represent the resolution of inflammation in the body (as it was described already), on the contrary, blood plasma 20-HETE may reflect dysregulation of inflammatory or metabolic processes. According to Li et al. ${ }^{36}, 20$-HETE plays a key role in insulin resistance, endothelial dysfunction, cardiovascular disease and diabetes pathogenesis. 20-HETE inhibit insulin-stimulated nitric oxide synthase (NOS) function and the production of nitric oxide (NO) in human umbilical vein endothelial cells (HUVECs). It is also associated with altered tyrosine phosphorylation of insulin receptor substrate-1 (IRS-1) and subsequent activation of PI3-kinase (PI3K), resulting in defective activation of Akt/eNOS signalling pathway ${ }^{36}$. Recently, Tunaru et al., showed that 20-HETE is an endogenous regulator of insulin secretion in pancreatic $\beta$-cells via a direct effect on Free fatty acid receptor 1 (FFAR1) receptors $^{10}$. Inhibition of 20-HETE production or FFAR1 receptor blockage was associated with a reduction in insulin secretion, and 20-HETE, in turn, was shown to be produced as a response to high glucose levels ${ }^{10}$.

We performed a multivariate binary regression analysis to identify factors that significantly affect the risk of high on-treatment platelet reactivity during dual antiplatelet therapy. According to Bonello et al., high on-treatment platelet reactivity in patients treated with antiplatelets after PCI, was defined as higher than $46 \%$ of maximal 5- $\mu \mathrm{mol} / \mathrm{l}$ ADP-induced aggregation ${ }^{17}$. Our results showed that insulin use and a higher 20-HETE concentration were all found to increase the risk of high on-treatment platelet reactivity. In contrast, ticagrelor use reduced the odds for high on-treatment platelet reactivity. In our patient sample, insulin was prescribed only to diabetic patients to control blood glucose levels. In clinical practice, insulin is prescribed to patients with type 1 diabetes, and also to patients with type 2 diabetes who are resistant to the action of insulin ${ }^{37}$. Diabetic patients usually have higher platelet reactivity, elevated oxidative stress and higher circulating free fatty acids (FFA), associated with chronic inflammation and a prothrombotic state $e^{8,9,38-40}$. Because of this, it is possible that insulin injection may have an effect on 20-HETE release.

Data from this study revealed that the efficiency of antiplatelet therapy may depend on such factors as insulin prescription and 20-HETE concentration. This study has some limitations. Firstly, the clinical factors may not be sufficiently reflected in our study. Blood glucose, glycated haemoglobin, and blood plasma lipoprotein levels were not analysed, and we did not evaluate the impact of CYP2C19 and CYP4F2 variants on the antiplatelet effect of drugs. Blood glucose levels, except patients with diabetes, are not routinely measured in our hospital. Patients with acute coronary syndromes usually have lower total cholesterol and high-density lipoprotein cholesterol levels. Low-density lipoprotein cholesterol also decreases during $\mathrm{MI}^{41}$. A significant portion of our described patients previously received lipid-lowering drugs. Thus, due to several factors, lipid profile might not be presented correctly in our studied patients.

\section{Conclusions}

Our results demonstrated that insulin use and 20-HETE level may determine high on-treatment platelet reactivity in patients receiving DAPT after ACS. The results of this study also showed that diabetes had no effect on antiplatelet treatment with ticagrelor, but it significantly reduced the antiplatelet activity of clopidogrel. Specific attention must be given to managing women with diabetes.

\section{Data availability}

The datasets and resources generated during and/or analyzed during the current study are available from the corresponding author upon reasonable request.

Received: 16 June 2019; Accepted: 17 January 2020;

Published online: 21 February 2020

\section{References}

1. Barbero, U. et al. Assessing Risk in Patients with Stable Coronary Disease: When Should We Intensify Care and Follow-Up? Results from a Meta-Analysis of Observational Studies of the COURAGE and FAME Era. Scientifica. 2016, 3769152 (2016).

2. Toth, P. P. Management of Acute Coronary Syndromes in Patients with Diabetes. J Fam Pract. 66, supp_az_1217 (2017).

3. Ibanez, B. et al. 2017 ESC Guidelines for the management of acute myocardial infarction in patients presenting with ST-segment elevation: The Task Force for the management of acute myocardial infarction in patients presenting with ST-segment elevation of the European Society of Cardiology (ESC). Eur. Heart J. 39, 119-177 (2018).

4. James, S. et al. Ticagrelor vs. clopidogrel in patients with acute coronary syndromes and diabetes: a substudy from the PLATelet inhibition and patient Outcomes (PLATO) trial. Eur. Heart J. 31, 3006-3016 (2010).

5. Castini, D. et al. Real-world clopidogrel utilization in acute coronary syndromes: patients selection and outcomes in a single-center experience. Ther. Adv. Cardiovasc. Dis. 11,323-331 (2017).

6. Schuette, C. et al. The effect of clopidogrel on platelet activity in patients with and without type-2 diabetes mellitus: a comparative study. Cardiovasc. Diabetol. 14, 15 (2015).

7. D’Ascenzo, F. et al. The prognostic impact of high on-treatment platelet reactivity with aspirin or ADP receptor antagonists: systematic review and meta-analysis. Biomed. Res. Int. 2014, 610296 (2014).

8. Maiocchi, S., Alwis, I., Wu, M. C. L., Yuan, Y. \& Jackson, S. P. Thromboinflammatory Functions of Platelets in Ischemia-Reperfusion Injury and Its Dysregulation in Diabetes. Semin. Thromb. Hemost. 44, 102-113 (2018).

9. Santilli, F., Simeone, P., Liani, R. \& Davì, G. Platelets and diabetes mellitus. Prostaglandins Other Lipid Mediat. 120, 28-39 (2015).

10. Tunaru, S. et al. S. 20-HETE promotes glucose-stimulated insulin secretion in an autocrine manner through FFAR1. Nat. Commun. 9, 177 (2018).

11. Wu, C. C., Gupta, T., Garcia, V., Ding, Y. \& Schwartzman, M. L. 20-HETE and blood pressure regulation: clinical implications. Cardiol. Rev. 22, 1-12 (2014).

12. Imig, J. D. \& Khan, A. H. Cytochrome P450 and Lipoxygenase Metabolites on Renal Function. Compr. Physiol. 6, 423-441 (2015). 
13. Kupstyte, N. et al. Effect of clinical factors and gene polymorphism of CYP2C19*2, *17 and CYP4F2*3 on early stent thrombosis. Pharmacogenomics. 16, 181-189 (2015).

14. Tatarunas, V., Kupstyte, N., Zaliunas, R., Giedraitiene, A. \& Lesauskaite, V. The impact of clinical and genetic factors on ticagrelor and clopidogrel antiplatelet therapy. Pharmacogenomics. 18, 969-979 (2017).

15. Tatarunas, V. et al. The impact of CYP2C19*2, CYP4F2*3, and clinical factors on platelet aggregation, CYP4F2 enzyme activity, and 20-hydroxyeicosatetraenoic acid concentration in patients treated with dual antiplatelet therapy. Blood Coagul. Fibrinolysis. 28, 658-664 (2017).

16. Steg, P. G. et al. ESC Guidelines for the management of acute myocardial infarction in patients presenting with ST-segment elevation. Eur. Heart J. 33, 2569-2619 (2012).

17. Bonello, L. et al. Consensus and future directions on the definition of high on-treatment platelet reactivity to adenosine diphosphate. J. Am. Coll. Cardiol. 56, 919-933 (2010).

18. Ferlini, M. et al. The paradox of clopidogrel use in patients with acute coronary syndromes and diabetes: insight from the Diabetes and Acute Coronary Syndrome Registry. Coron. Artery Dis. 29, 309-315 (2018).

19. Harding, S. A. et al. Contemporary antiplatelet therapy in acute coronary syndromes: are there differences in outcomes and discontinuation between clopidogrel and ticagrelor? Intern. Med. J. 47, 1298-1305 (2017).

20. Núñez, J. et al. Low lymphocyte count in acute phase of ST-segment elevation myocardial infarction predicts long-term recurrent myocardial infarction. Coron. Artery Dis. 21, 1-7 (2010).

21. Fazlinezhad, A. et al. The Relationship between Serum Hemoglobin and Creatinine Levels and Intra-Hospital Mortality and Morbidity in Acute Myocardial Infarction. Int. Cardio Res. J. 9, e10912 (2017).

22. Nardin, M. et al. Diabetes mellitus, glucose control parameters and platelet reactivity in ticagrelor treated patients. Thromb. Res. 143, 45-49 (2016)

23. Wallentin, L. et al. Effect of CYP2C19 and $\mathrm{ABCB} 1$ single nucleotide polymorphisms on outcomes of treatment with ticagrelor versus clopidogrel for acute coronary syndromes: a genetic substudy of the PLATO trial. Lancet. 376, 1320-1328 (2010).

24. Teng, R., Oliver, S., Hayes, M. A. \& Butler, K. Absorption, distribution, metabolism, and excretion of ticagrelor in healthy subjects. Drug. Metab. Dispos. 38, 1514-1521 (2010).

25. Shuldiner, A. R. et al. Association of cytochrome P450 2C19 genotype with the antiplatelet effect and clinical efficacy of clopidogrel therapy. JAMA. 302, 849-857 (2009).

26. Trenk, D. \& Hochholzer, W. Genetics of platelet inhibitor treatment. Br. J. Clin. Pharmacol. 77, 642-653 (2014).

27. Bonello, L. et al. Ticagrelor increases adenosine plasma concentration in patients with an acute coronary syndrome. J. Am. Coll. Cardiol. 63, 872-877 (2014).

28. Sato, A. et al. Mechanism of vasodilation to adenosine in coronary arterioles from patients with heart disease. Am. J. Physiol. Heart Circ. Physiol. 288, H1633-H1640 (2005).

29. Harvey, R. D. \& Morgan, E. T. Cancer, inflammation, and therapy: effects on cytochrome p450-mediated drug metabolism and implications for novel immunotherapeutic agents. Clin. Pharmacol. Ther. 96, 449-457 (2014).

30. Morgan, E. T. Regulation of cytochrome p450 by inflammatory mediators: why and how? Drug. Metab. Dispos. 29, 207-212 (2001).

31. Johnson, A. L., Edson, K. Z., Totah, R. A. \& Rettie, A. E. Cytochrome P450 w-Hydroxylases in Inflammation and Cancer. Adv. Pharmacol. 74, 223-262 (2015).

32. Alvarellos, M. L. et al. PharmGKB summary: very important pharmacogene information for CYP4F2. Pharmacogenet Genomics. 25, 41-47 (2015)

33. Hirani, V., Yarovoy, A., Kozeska, A., Magnusson, R. P. \& Lasker, J. M. Expression of CYP4F2 in human liver and kidney: assessment using targeted peptide antibodies. Arch. Biochem. Biophys. 478, 59-68 (2008).

34. Kanemoto, S. et al. Multivesicular body formation enhancement and exosome release during endoplasmic reticulum stress. Biochem. Biophys. Res. Commun. 480, 166-172 (2016).

35. Powell, P. K., Wolf, I., Jin, R. \& Lasker, J. M. Metabolism of arachidonic acid to 20-hydroxy-5,8,11, 14-eicosatetraenoic acid by P450 enzymesin human liver: involvement of CYP4F2 and CYP4A11. J. Pharmacol. Exp. Ther. 285, 1327-1336 (1998).

36. Li, X. et al. 20-Hydroxyeicosatetraenoic acid impairs endothelial insulin signaling by inducing phosphorylation of the insulin receptor substrate-1 at Ser616. PLoS One. 9, e95841 (2014).

37. Goldstein, B. J. \& Dirk, M. W. Type 2 diabetes: principles and practice. (CRC Press, 2016).

38. Santilli, F. et al. Microparticles as new markers of cardiovascular risk in diabetes and beyond. Thromb. Haemost. 116, 220-234 (2016).

39. Kashyap, S. et al. A sustained increase in plasma free fatty acids impairs insulin secretion in nondiabetic subjects genetically predisposed to develop type 2 diabetes. Diabetes. 52, 2461-2474 (2003)

40. Hara, T., Hirasawa, A., Ichimura, A., Kimura, I. \& Tsujimoto, G. Free fatty acid receptors FFAR1 and GPR120 as novel therapeutic targets for metabolic disorders. J. Pharm. Sci. 100, 3594-3601 (2011).

41. Hubacek, J. A. et al. Traditional risk factors of acute coronary syndrome in four different male populations - total cholesterol value does not seem to be relevant risk factor. Physiol. Res. 66, S121-S128 (2017).

\section{Acknowledgements}

We thank Julija Vasilenko-Maskvyte for editorial help. These studies were funded by a grant (No. SEN-09/2015) from the Research Council of Lithuania.

\section{Author contributions}

V.T., N.K.K. and V.J. conceived, designed, conducted the experiment, analysed the data, wrote and revised the manuscript. R.Z. and V.L. wrote and revised the manuscript. V.Z. conducted the experiments and analysed the data.

\section{Competing interests}

The authors declare no competing interests.

\section{Additional information}

Correspondence and requests for materials should be addressed to V.T.

Reprints and permissions information is available at www.nature.com/reprints.

Publisher's note Springer Nature remains neutral with regard to jurisdictional claims in published maps and institutional affiliations. 
(c) (i) Open Access This article is licensed under a Creative Commons Attribution 4.0 International License, which permits use, sharing, adaptation, distribution and reproduction in any medium or format, as long as you give appropriate credit to the original author(s) and the source, provide a link to the Creative Commons license, and indicate if changes were made. The images or other third party material in this article are included in the article's Creative Commons license, unless indicated otherwise in a credit line to the material. If material is not included in the article's Creative Commons license and your intended use is not permitted by statutory regulation or exceeds the permitted use, you will need to obtain permission directly from the copyright holder. To view a copy of this license, visit http://creativecommons.org/licenses/by/4.0/.

(C) The Author(s) 2020 